\title{
O.S.P.
}

L'orientation scolaire et professionnelle

$30 / 3 \mid 2001$

Juger et être jugé : perspectives psychosociales

\section{Juger et être jugé : présentation}

To judge and to be judged: an introduction

\section{Pascal Pansu}

\section{OpenEdition}

Journals

Édition électronique

URL : http://journals.openedition.org/osp/5089

DOI : 10.4000/osp.5089

ISSN : 2104-3795

Éditeur

Institut national d'étude du travail et d'orientation professionnelle (INETOP)

Référence électronique

Pascal Pansu, « Juger et être jugé : présentation », L'orientation scolaire et professionnelle [En ligne], 30/3 | 2001, mis en ligne le 15 septembre 2004, consulté le 16 décembre 2020. URL : http:// journals.openedition.org/osp/5089; DOI : https://doi.org/10.4000/osp.5089

Ce document a été généré automatiquement le 16 décembre 2020.

(c) Tous droits réservés 


\title{
Juger et être jugé : présentation
}

\author{
To judge and to be judged: an introduction
}

\section{Pascal Pansu}

1 Les termes juger ${ }^{1}$ et évaluer font, l'un comme l'autre, partie du vocabulaire usuel en psychologie, et en tant qu'objet d'étude tendent même à y occuper une place importante. Bien que souvent employés dans des acceptations voisines et présentant la caractéristique d'être substituables, la question de la différence de dénotation entre ces deux termes mérite d'être soulevée dans cette partie introductive. Sans pour autant prétendre à établir l'univocité de chacun de ces termes ou encore à les définir de manière exhaustive, il convient de préciser une des raisons principales qui nous a conduit à préférer le mot juger à celui d'évaluer. Cette raison tient, tout simplement, dans l'usage que l'on fait couramment de ces termes.

2 Le mot évaluation a pris dans le vocabulaire courant un sens de plus en plus techniciste. Il en est pour preuve, l'intitulé des actes du Congrès International de Paris en 1993, « les techniques psychologiques d'évaluation des personnes » (Huteau, 1993). Dans ses usages les plus courants, l'évaluation est donc décrite comme un ensemble de connaissances et de techniques qui permettent de répondre à toute une série de demandes sociales d'une société technicienne dont la sélection (via les examens, les diplômes, etc.) est l'assise. En ce sens, l'évaluation est un rouage essentiel pour assurer la stabilité du système social, "rouage essentiel, en ce que l'évaluation est présente dans tout acte de perception, d'orientation, d'affectation ou de promotion » (Beauvais, 1976, p. 3). Sans doute est-ce aussi pour répondre aux mieux à de telles exigences sociales qu'on voit parfois des professionnels préoccupés d'évaluation, se comporter comme des maniaques de l'évaluation technique, des obsédés des outils de mesure, et ce dans l'unique but de légitimer le diagnostic posé sur autrui et les décisions qui engagent son avenir. Autrement dit, la fortune techniciste du mot évaluation résulte très probablement des discours sur l'évaluation où l'individu est appréhendé du point de vue de sa dépendance et de son adéquation psychologique à l'organisation. Ainsi, quoique appréhendable en tant qu'objet d'étude de la psychologie à plusieurs niveaux, " celui des processus, celui des pratiques ou encore celui des techniques et méthodes " (Monteil, 1990, pp. 51-52), l'évaluation semble surtout caractérisée sous un aspect 
techniciste. A ce titre, le terme évaluer est donc surtout employé en référence aux différentes mesures effectuées en psychologie (i.e. psychométrie), à leur signification, à leur validité empirique, voire également à d'autres champs d'étude dont l'objet est d'approcher par le biais des mesures une « objectivité » scientifique qui parait aller de soi (Voir à ce propos la critique qu'en fait De Montmollin (1972), dans son célèbre ouvrage, Les psychopitres, qui reste pour le moins dans l'air du temps). l'idée de sanctions formelles positives (récompenses) ou négatives (punition). Si les sanctions formelles, en tant que réponses aux atteintes à la règle prescrite par la loi, sont à ce point saillantes dans la société, les sanctions auxquelles nous sommes le plus souvent confrontées dans notre vie quotidienne sont d'ordre informelles. Par exemple, le prestige ou la mauvaise réputation dont bénéficie Harvey dans son entourage constitue, dans un cas comme dans l'autre, une sanction fortement significative. En avançant qu'Harvey est quelqu'un de particulièrement doué ou de particulièrement incapable, nous portons bien là un jugement sur la valeur sociale et l'utilité de Harvey, plus que nous ne les mesurons. Ainsi, dans notre quotidien, nous nous comportons plus souvent comme des juges du comportement d'autrui que comme des scientifiques cherchant à éprouver les caractéristiques complexes du comportement d'autrui. Le professionnel de l'évaluation, qu'il exerce dans le domaine scolaire (instituteurs, professeurs, formateurs, etc.) ou celui de l'entreprise (responsables des ressources humaines, cadres hiérarchiques, etc.) n'échappe pas pour autant à un tel comportement social. Pas plus que tout un chacun, il n'est un psychométricien. En ce sens, ce n'est pas quelqu'un qui mesure, mais bien plutôt une personne qui fournit une connaissance générale ou généralisée sur autrui. 11 juge plus qu'il ne mesure.

Dans cette optique, l'activité évaluative scolaire ou professionnelle n'est pas foncièrement différente du jugement quotidien qu'on porte sur autrui : les mécanismes auxquels elle obéit sont semblables, seul l'objet du jugement change. L'évaluation passe donc par une conduite sociale qui consiste à juger de la valeur d'une personne en référence à l'utilité des comportements ou des résultats qu'elle nous renvoie. C'est pour insister sur le fait que cette activité d'évaluation ne se réduit pas à une simple mesure des habiletés individuelles, que la notion de jugement social nous est apparue ici plus appropriée que celle d'évaluation dans le choix de l'intitulé de ce numéro.

L'idée de jugement social est d'ailleurs loin d'être récente. Les psychologues sociaux l'ont développé depuis maintenant plus de 50 ans. C'est même l'un des objets majeurs de la psychologie sociale depuis la parution du livre de Heider (1958) « The psychology of interpersonal relation » qui a tant marqué le développement de cette discipline aux ÉtatsUnis. Heider, en analyste de la psychologie de tous les jours, y développe la notion d'attribution. Cette notion dénote les processus qui permettent de donner du sens aux événements auxquels nous sommes confrontés : i.e. comment les gens expliquent et interprètent les événements auxquels ils sont confrontés dans leur vie quotidienne, et notamment les comportements d'autrui. D'autres psychologues sociaux français ont situé les pratiques du jugement dans leur contexte social et institutionnel. C'est le cas de Beauvois qui, dès 1976, analyse les conséquences épistémologiques et cognitives de la place qu'ont les conduites d'évaluation dans ce contexte. Cela le conduit à voir la genèse du modèle des différences individuelles (si aisément technologisable) dans le fait purement social de la remplaçabilité des personnes. L'idée défendue par Beauvois est que les pratiques évaluatives sont constitutives de la vie sociale et des structures 
sociales. Sans entrer dans les métaphores du monde animal, si on trouve de la domination et des échanges dans les sociétés animales (dans la meute le loup, qu'il soit rabatteur ou chasseur, sait qu'il aura sa part du butin), on ne trouve pas d'agents mandatés pour en juger d'autres afin d'organiser leur remplaçabilité (même chez les singes, pourtant les plus proches de l'homme). Le jugement et son institutionnalisation dans les conduites d'évaluation seraient donc, en ce sens, la caractéristique fondatrice du social humain.

6 Notre objectif, on l'aura compris, n'est pas d'aborder les aspects technicistes qu'évoque l'évaluation. Il s'agit d'apporter des éléments susceptibles d'éclairer le lecteur - à partir de quelques contributions marquées par une orientation psychosociale - à la fois, sur les pratiques et mécanismes qui déterminent l'acte à partir duquel le jugement est émis et également sur les stratégies d'autoprésentation qui permettraient aux personnes jugées d'être bien perçues.

7 Le texte de Dépret et Filisetti passe en revue des apports de recherches en psychologie sociale concernant le problème du jugement social et dégage des pistes de réflexion et d'action pour les professionnels de l'éducation. Les auteurs montrent, d'une part, comment les psychologues sociaux mettent en garde l'évaluateur contre les nombreux biais socio-cognitifs et socio-affectifs qui affectent les jugements qu'on porte sur autrui. D'autre part, ils suggèrent que, bien que les psychologues sociaux se soient surtout intéressés à la source du jugement, il est utile de prendre en compte la cible du jugement et l'interaction entre la cible et la source. De ce point de vue, la notion de compétence sociale s'avère pour Dépret et Filisetti une notion essentielle pour une meilleure compréhension des processus du jugement social.

Nous retrouvons cette notion de compétence (ou d'habileté) sociale dans la recherche empirique décrite par Joly-Pottuz, Desrichard et Carbonnel. Elle concerne les difficultés que rencontrent des personnes ayant des troubles cognitifs résultant d'un traumatisme crânien grave dès lors qu'il s'agit de s'autoprésenter dans une situation très normative. Une des caractéristiques de ces patients est qu'ils ont des difficultés à mettre en place des stratégies $d$ ' autoprésentation qui leur permettraient d'être bien perçus. Les auteurs défendent l'intérêt d'une prise en charge adaptée qui devrait, via l'apprentissage de compétences en matière de stratégies d'autoprésentation, leur permettre de mieux s'intégrer dans leur environnement social et professionnel.

9 Cette notion d'auto-présentation est centrale dans le concept de clairvoyance normative que nous présente Guingouain. Cet auteur décrit la clairvoyance normative comme une compétence (ou habileté) sociale à reconnaître l'aspect valorisé de certaines conduites ou attitudes, compétences qui, en retour, permet à l'individu d'ajuster aux mieux ses comportements selon les objectifs qu'il veut atteindre. Guingouain envisage, ici, la clairvoyance normative dans une perspective métacognitive et présente cette compétence (ou habileté) comme nécessaire à une insertion sociale réussie. Après avoir exposé les résultats d'une étude empirique qui supporte cette hypothèse, l'auteur discute des implications pédagogiques d'une telle compétence sociale.

10 L'étude conduite par Bressoux et Pansu rejoint les travaux sur la clairvoyance normative et examine comment s'élabore la construction du jugement scolaire. Les résultats confirment tout d'abord l'idée selon laquelle le jugement que l'enseignant porte sur un élève ne se fonde pas uniquement sur le niveau individuel des performances de cet élève, mais également sur le niveau des autres élèves de la classe 
(effet de contexte). Ils montrent ensuite que lorsqu'il s'agit de donner une bonne image de soi, l'internalité est liée positivement et linéairement au jugement de l'enseignant. Ce résultat semble traduire l'effet que peut avoir la clairvoyance de certains élèves quant au caractère valorisant des explications internes. On peut voir là, une véritable habileté sociale qui s'exprime dans l'ajustement d'une attitude à une attente sociale. Curieusement, l'attitude qu'on pourrait croire symétrique, qui consiste à se montrer externe pour se faire mal voir, ne produit aucun effet significatif sur le jugement de l'enseignant. Cette non-symétrie incite Bressoux et Pansu à discuter de la notion de clairvoyance telle qu'elle est abordée traditionnellement (voir le rappel que fait Guinguouain dans son article).

11 L'étude conduite par Cyril Tarquinio et Pascale Tarquinio, concerne l'influence des stéréotypes d'attractivité, d'une part, et des biais socio-cognitifs, d'autre part, sur les jugements des enseignants et leurs décisions de passage d'un élève dans une classe supérieure. Les élèves cibles des décisions sont des élèves moyens/faibles scolairement, connus aussi pour être plus ou moins attractifs (photographie) et normatifs (questionnaire d'internalité). Les résultats obtenus peuvent paraître surprenants : lorsque les élèves sont moyens, les auteurs observent un effet positif de l'attractivité, mais ce uniquement lorsqu'il s'agit d'élèves connus pour avoir choisi des explications externes. Cet effet disparaît lorsqu'il s'agit d'élèves connus pour avoir choisi des explications internes. En revanche, lorsque les élèves sont moyens/faibles les résultats sont différents : les élèves les moins attractifs sont toujours favorisés qu'ils soient ou non internes.

12 Le texte de Dubois et Beauvois présente d'abord des recherches qui montrent l'existence de deux composantes de la valeur des personnes. La première composante correspond au concept de désirabilité et renvoie au registre affectif et émotionnel (i.e. ce qu'on ressent, ce qu'on aime et ce qu'on n'aime pas, etc.). La seconde composante de la valeur correspond aux utilités sociales (i.e. ce qui est utile dans la vie sociale) et renvoie à un registre qu'on peut qualifier de quasi économique. Les auteurs présentent ensuite d'autres recherches pour montrer que la position de juge ou d'évaluateur prédispose à la prise en compte de la seconde de ces composantes. L'utilité serait, selon eux, davantage associée à la normativité des jugements que l'on porte sur la valeur d'autrui parce qu'elle fournit d'emblée des informations sur ce que l'on peut attendre ou faire d'autrui dans un contexte institutionnel ou de travail.

Enfin Curie, en chercheur averti sur l'effet et l'impact des biais socio-affectifs et sociocognitifs qui affectent les jugements qu'on porte sur autrui, nous propose une lecture critique des différentes contributions de ce numéro.

Puisse le lecteur y trouver, à défaut de recettes, quelques éléments de réflexions utiles et susceptibles de l'éclairer sur les fondements et l'utilisation des pratiques sociales de jugement. 


\section{BIBLIOGRAPHIE}

Beauvois, J.-L. (1976). Problématique des conduites sociales d'évaluation. Connexions, 19, 7-30. Heider, F. (1958). The psychology of interpersonal relation. New York : Wiley.

Monteil, J.-M. (1990). L'évaluation scolaire : fragments de recherches en psychologie. Connexions, $56,51-66$.

Montmollin (De), M. (1972). Les psychopitres. Paris : P.U.F.

Huteau, M. (1993). Les techniques psychologiques d'évaluation des personnes. Collection Sciences Humaines. E.A.P. : Paris.

\section{NOTES}

1. Ce numéro a été coordonné par Pascal Pansu, Université de Savoie Chambéry, Département de Psychologie et membre du L.P.S., route du Sergent Revel BP 1104, 73000 Chambéry. Courriel Pascal.Pansu @ univ-savoie

\section{AUTEUR}

\section{PASCAL PANSU}

est Maître de Conférences en Psychologie Sociale à l'Université de Savoie Chambéry, membre de l'équipe de recherche du Laboratoire de Psychologie Sociale Grenoble-Chambéry (L.P.S.). Ses recherches récentes portent sur le jugement social, plus particulièrement sur l'impact des normes de jugement comme la norme d'internalité, la construction du jugement scolaire et ses effets sur la perception de soi des élèves, en particulier leur l'impact sur le sentiment de compétence scolaire. 